\title{
Development of patient-derived tumor organoids and a drug testing model for renal cell carcinoma
}

\author{
AKIRA KAZAMA $^{1}$, TSUTOMU ANRAKU ${ }^{1}$, HIROO KUROKI ${ }^{1}$, YUKO SHIRONO ${ }^{1}$, \\ MASAKI MURATA ${ }^{1}$, VLADIMIR BILIM ${ }^{1,2}$, ANDREY UGOLKOV ${ }^{3}$, \\ KAZUHIDE SAITO $^{1}$ and YOSHIHIKO TOMITA ${ }^{1}$ \\ ${ }^{1}$ Department of Urology, Division of Molecular Oncology, Niigata University Graduate School of \\ Medical and Dental Sciences, Niigata 951-8510; ${ }^{2}$ Kameda Daiichi Hospital, Niigata 950-0165, \\ Japan; ${ }^{3}$ Actuate Therapeutics, Inc., Fort Worth, TX 76107, USA
}

Received April 1, 2021; Accepted July 26, 2021

DOI: $10.3892 /$ or.2021.8177

\begin{abstract}
The selection of effective therapeutic agents is critical for improving the survival of patients with renal cell carcinoma (RCC). The aim of the present study was to develop an ex vivo drug testing assay using patient-derived tumor organoid (TO) cultures. For this purpose, surgical tumor specimens were obtained from 20 patients with RCC. TOs were developed ex vivo from freshly resected RCC tumors, and their histopathological and molecular characteristics were evaluated using histological staining and whole-exome sequencing (WES). Using a cell viability assay, the therapeutic efficacy of standard of care tyrosine kinase inhibitors in RCC TOs was determined. It was found that TOs recapitulated the histological features of primary RCC tumors. Using WES, a strong concordance was identified at the genetic level between the primary tumors and their corresponding TOs. Using patient-derived TO models, a prototype of an ex vivo drug testing assay was developed, and it was found that RCC TOs exhibited differential responses to sunitinib, pazopanib, cabozantinib, axitinib and sorafenib treatment. On the whole, although the predictive value of the current assay has to be tested and validated in future clinical studies, the findings of the present study demonstrate a novel approach for ex vivo drug testing in patient-derived TO models, which may have potential for use in the personalized treatment of cancer patients.
\end{abstract}

Correspondence to: Professor Yoshihiko Tomita, Department of Urology, Division of Molecular Oncology, Niigata University Graduate School of Medical and Dental Sciences, 1-757 Asahimachi-dori, Chuo-ku, Niigata 951-8510, Japan

E-mail: y.tomita@med.niigata-u.ac.jp

Key words: tumor organoid, renal cell carcinoma, personalized therapy, precision medicine

\section{Introduction}

Renal cell carcinoma (RCC) accounts for $\sim 3-5 \%$ of all carcinomas. According to the World Health Organization, $>140,000$ individuals succumb to the disease annually (1). Systemic treatment is required for $25 \%$ of patients with RCC with metastases detected at the time of initial diagnosis and for $>10 \%$ of patients with RCC experiencing recurrence following the surgical resection of the tumor (2). The personalized selection of the most effective drugs is essential to improve the survival of patients with advanced RCC. In recent years, the selection of treatment strategies for metastatic RCC has become increasingly complex with the advent of targeted therapeutics and immune checkpoint inhibitors (ICIs). Currently, only $10 \%$ of all cancer patients have actionable targets (detected by next-generation sequencing, immunohistochemical staining and other bioanalytical methods) that can be used to select targeted therapeutics (3). Owing to tumor heterogeneity and the complexity of cancer signaling networks, the personalized functional assessment of each tumor may be more valuable than the analysis of tumor molecular profiles. Recent advances in the development of patient-derived tumor organoids (TOs) have provided new opportunities for the growth and analysis of patient tumors ex vivo. TOs are three-dimensional (3D) tissue-like cancer cell clusters derived from tumor tissue that mimic the in vivo characteristics and cellular heterogeneity of the original tumor (4-6). Two-dimensional (2D) cancer cell lines have been used in cancer research for decades. However, the generation of 2D cell lines from human tumors is highly inefficient, as it requires the lengthy selection of cancer cells for $2 \mathrm{D}$ culture conditions and numerous passages of rare clones in 2D cultures, with potential genetic changes leading to the establishment of genetically monoclonal cell lines that do not represent the genetic heterogeneity of the original tumor (7). On the other hand, the 3D growth of TOs resembles clinical tumors; moreover, TOs preserve the heterogeneity of the parental tumors. Thus, TOs have emerged as a potential ex vivo tumor model for the development of personalized therapy $(8,9)$. Clinical tumors and patient-derived TO cultures share a number of features, including morphology, cell-cell interaction, signal transduction, gene and protein 
expression $(10,11)$. Recent studies have demonstrated that the therapeutic response of patient-derived TOs to antitumor drugs ex vivo may be promising for the development of personalized treatment (10-12).

The present study reports the development of a novel method for the efficient establishment of patient-derived TO cultures from RCC tumor samples. It is demonstrated that RCC TOs recapitulate the histological features of the clinical tumor ex vivo and maintain the genomic features of their originating tumors during long-term culturing. Using a panel of RCC standard of care (SOC) tyrosine kinase inhibitors (TKIs) and patient-derived RCC TOs, an ex vivo testing method was developed for potential use in customizing and tailoring RCC treatment for individual patients.

\section{Materials and methods}

Development of patient-derived TOs. The present study was approved by the Institutional Review Board of Niigata University (approval no. 2018-0254). Written informed consent was obtained from all patients. Tumor samples were collected from RCC tissues obtained via radical nephrectomy or nephron-sparing surgery from 20 patients at Niigata University Hospital between 2018 and 2020. The patient characteristics are presented in Table I.

The TO culture conditions were based on those previously reported in the study by Lee et al (13) in prostate and bladder cancer models, including Matrigel (\#354230, Corning, Inc.) to support 3D TO culture, hepatocyte medium and Rho-associated protein kinase (ROCK) inhibitors. The step-by-step procedure of tissue processing and development of TO culture is illustrated in Fig. 1. The tumor samples were $1 \times 1 \mathrm{~cm}$ tissue blocks obtained from the portion of the kidney tumor removed in the operating room, which were promptly cooled and transported to the laboratory for processing. A portion of the sample was preserved as a frozen specimen and used for DNA collection. For tissue dissociation, RCC tissues were placed in cold phosphate-buffered saline (PBS) and minced using scalpels. The tumor tissue was incubated in $2 \mathrm{ml}$ of collagenase solution (\#07912, STEMCELL Technologies) at $37^{\circ} \mathrm{C}$ for $30 \mathrm{~min}$. The dissociated tissue was spun down (200 x g at room temperature for $5 \mathrm{~min}$ ), resuspended in PBS, and passed through a 100- $\mu$ m cell strainer (\#435010003, Funakoshi Co., Ltd.). The cells were then spun down $(200 \mathrm{x} g$ at room temperature for $5 \mathrm{~min}$ ), gently mixed with Matrigel on ice, and placed in a 24 -well plate. Following a $20-\mathrm{min}$ at $37^{\circ} \mathrm{C}$ incubation to solidify the Matrigel, the TO culture medium was added and the cells were incubated at $37^{\circ} \mathrm{C}$.

Histological analysis and immunohistochemical (IHC) staining. The TOs were fixed in $10 \%$ formalin and embedded in paraffin. The paraffin-embedded sections (4- $\mu \mathrm{m}$-thick) were prepared and stained with hematoxylin and eosin using standard protocols. IHC staining was performed as previously described (14). 1/100 dilution anti-B-cell lymphoma 2 (Bcl-2) mouse monoclonal antibody (\#MAB11332, Abnova) was used for IHC staining (60 min at room temperature). After washing with PBS, the slides were incubated with horseradish peroxidase (HRP)-labeled secondary antibody for $30 \mathrm{~min}$ at room temperature (Histofine simple stain MAX-PO,
\#424152, Nichirei). The staining reaction was developed using 3,3'-diaminobenzidine (DAB), and nuclear counterstaining was performed with hematoxylin (Mayer's Hematoxylin Solution, \#131-09665, FUJIFILM Wako Pure Chemical Corporation) for $5 \mathrm{~min}$ at room temperature. The sections were analyzed under an Olympus DP72 microscope (Olympus Corporation).

Whole-exome sequencing (WES). To identify somatic mutations in patient-derived TOs and parental tumor tissues, WES was performed. Genomic DNA from each sample was used to construct a paired-end sequencing library. The DNA quality was evaluated using $1 \%$ agarose gel electrophoresis and a NanoDrop spectrophotometer (Thermo Fisher Scientific, Inc.). The samples were prepared using the SureSelect Human All Exon 50Mb kit (Agilent Technologies, Inc.). WES analysis was performed using an Illumina HiSeq2500 instrument (Illumina). The informatics analysis, mainly including quality control, read mapping, variant calling, filtering and annotation, was conducted using BWA software (http://bio-bwa. sourceforge.net/bwa.shtml), GATK (https:/www.broadinstitute.org/gatk/) and the SnpEff tool (http://snpeff.sourceforge. net/SnpEff.html), respectively.

Cell viability assay and TKIs. The TOs were grown in flat-bottom 96-well plates (Corning, Inc.) at $37^{\circ} \mathrm{C}$ in a $5 \% \mathrm{CO}_{2}$ atmosphere and treated with various concentrations $(0.1,0.5,1,5,10,50,100$ and $500 \mu \mathrm{M})$ sunitinib (\#PZ0012, Sigma-Aldrich; Merck KGaA), pazopanib (\#12097, Cayman Chemical Company), cabozantinib (\#C8999, LC Laboratories), axitinib (\#PZ0193, Sigma-Aldrich; Merck KGaA) and sorafenib (\#CS0164, Chemscene) for $72 \mathrm{~h}$. TO proliferation was measured using a CellTiter $96^{\circledR}$ AQueous One Solution Cell Proliferation assay (\#93582, Promega Corporation). The experiments were repeated three times, and the results were read using an iMark ${ }^{\mathrm{TM}}$ 96-well microplate reader (Bio-Rad Laboratories, Inc.). The absorbance was measured at $490 \mathrm{~nm}$. The drug concentration that inhibited the growth of cancer cells by $50 \%\left(\mathrm{GI}_{50}\right)$ for each drug was calculated using GraphPad Prism software (version 8.0; GraphPad Software, Inc.).

\section{Results}

Histopathological features of RCC tumors and tumor-derived TOs. The present study developed TO organoid cultures from freshly resected human tumors in 15 of 20 RCC cases (Table I). The histopathological diagnosis was clear cell carcinoma in all tumors, apart from one case of chromophobe RCC. Approximately half of the RCC cases (9/20) had a Fuhrman grade $\geq 3$. The established RCC TOs were propagated for three or more passages and cryopreserved. Although TO culture was established in the majority of cases, a cessation of TO growth was observed after several passages in 5 of the 20 RCC cases (Table I). The established TOs were cultured for up to 15 passages over a 12 -month period. No apparent loss of growth capacity was observed following multiple passages, and all 15 established TO cultures were successfully restarted following cryopreservation. The cultured TOs were passaged every 3-4 weeks. The TOs grew in dense clusters of cells, forming aggregates. A high degree of association between 
Table I. Analysis of RCC clinicopathological parameters of RCC cases and corresponding tumor organoids.

\begin{tabular}{|c|c|c|c|c|c|c|c|c|c|}
\hline Case & $\begin{array}{l}\text { Age, } \\
\text { years }\end{array}$ & Sex & Pathology & $\begin{array}{l}\text { ISUP } \\
\text { grade }\end{array}$ & $\begin{array}{l}\text { Fuhrman } \\
\text { grade }\end{array}$ & T stage & $\begin{array}{c}\text { TO } \\
\text { Growth }\end{array}$ & Pre Tx & Post Tx \\
\hline OR007 & 65 & $\mathrm{M}$ & Clear cell & G2 & G2 & pT1a & $+/+$ & - & - \\
\hline OR008 & 46 & M & $\begin{array}{l}\text { Clear cell + } \\
\text { spindle cell } \\
\text { component }\end{array}$ & G4 & G4 & pT1b & $+/+$ & - & $\begin{array}{l}\text { Ipi }+ \\
\text { Nivo }\end{array}$ \\
\hline OR009 & 29 & $\mathrm{M}$ & $\begin{array}{l}\text { Chromophobe + } \\
\text { sarcomatoid diff }\end{array}$ & G4 & G4 & pT3a & $+/+$ & $\begin{array}{l}\text { Ipi }+ \\
\text { Nivo }\end{array}$ & - \\
\hline OR010 & 77 & M & $\begin{array}{l}\text { Clear cell + } \\
\text { sarcomatoid }\end{array}$ & G2 & $\mathrm{G} 2$ & pT1a & $+/+$ & - & - \\
\hline OR011 & 68 & $\mathrm{~F}$ & $\begin{array}{l}\text { Clear cell + } \\
\text { sarcomatoid + } \\
\text { rhabdoid diff }\end{array}$ & G4 & G4 & pT3a & $+/+$ & Axi & Axi \\
\hline OR012 & 73 & M & Clear cell & G2 & G2 & pT1b & $+/+$ & - & - \\
\hline OR013 & 72 & M & Clear cell & G2 & $\mathrm{G} 2$ & pT1b & $+/+$ & - & - \\
\hline OR014 & 53 & M & Clear cell & G2 & G2 & pT1b & $-/-$ & - & - \\
\hline OR015 & 71 & $\mathrm{M}$ & Clear cell & G4 & G4 & pT1a & $+/-$ & - & - \\
\hline OR016 & 81 & M & $\begin{array}{l}\text { Cear cell + } \\
\text { sarcomatoid + } \\
\text { rhabdoid diff }\end{array}$ & G4 & G4 & pT3a & $+/-$ & - & - \\
\hline OR017 & 50 & $\mathrm{~F}$ & Clear cell & G2 & $\mathrm{G} 2$ & pT1a & $-/-$ & - & - \\
\hline OR018 & 52 & M & $\begin{array}{l}\text { Clear cell + } \\
\text { sarcomatoid + } \\
\text { rhabdoid diff }\end{array}$ & G4 & G4 & pT4 & $+/+$ & $\begin{array}{c}\text { Pem }+ \\
\text { Axi }\end{array}$ & $\begin{array}{c}\mathrm{Paz} \rightarrow \\
\text { Nivo }\end{array}$ \\
\hline OR019 & 48 & $\mathrm{~F}$ & Clear cell & $\mathrm{G} 2$ & G2 & pT1a & $+/+$ & - & - \\
\hline OR020 & 78 & M & Clear cell & G2 & $\mathrm{G} 2$ & pT2b & $+/+$ & - & - \\
\hline OR021 & 39 & M & Clear cell & G2 & $\mathrm{G} 2$ & pT1a & $+/+$ & - & - \\
\hline OR022 & 69 & $\mathrm{M}$ & $\begin{array}{l}\text { Clear cell + } \\
\text { rhabdoid diff }\end{array}$ & G4 & G4 & pT3 & $+/+$ & - & - \\
\hline OR023 & 76 & M & Clear cell & G4 & G4 & pT4 & $+/+$ & - & - \\
\hline OR024 & 76 & M & Clear cell & G2 & G2 & pT1b & $+/+$ & - & - \\
\hline OR025 & 77 & $\mathrm{~F}$ & $\begin{array}{l}\text { Clear cell + } \\
\text { sarcomatoid }\end{array}$ & $\mathrm{G} 3>\mathrm{G} 4$ & $\mathrm{G} 3>\mathrm{G} 4$ & pT1b & $+/-$ & - & - \\
\hline OR026 & 72 & $\mathrm{M}$ & Clear cell & G1 & $\mathrm{G} 2$ & pT3a & $+/+$ & - & - \\
\hline
\end{tabular}

TO, tumor organoids; ISUP, International Society of Urological Pathology; Tx, treatment; diff, differentiation; Ipi, ipilimumab; Nivo, nivolumab; Pem, pembrolizumab; Axi, axitinib; Paz, pazopanib; +/+, growth and propagation; +/-, only growth, no propagation; -/-, no growth.

the morphological structure of RCC tumors and their corresponding TOs was observed (Fig. 2). The present study not only confirmed the histology of clear cells, a common histological feature of RCC in TOs, but also the features of chromophobe $\mathrm{RCC}$ and renal carcinoma with sarcomatoid variants. Using IHC staining, it was found that $\mathrm{Bcl}-2$ was highly expressed in cancer cells from both RCC tissues and the corresponding TOs (Fig. 3).

WES of renal tumors and tumor-derived TOs. To investigate whether the TOs preserved the genetic characteristics of their corresponding RCC tumors, WES of the TOs and their corresponding primary tumor tissues was performed using a NovaSeq 6000 system. The allele frequencies of the variant genes represented the multiclonal capacity of a TO culture compared to parental cancer tissue. Although 6-39\% of the allele settings differed between the tumor tissues and TOs, the analysis of the WES data revealed concordance in numerous gene mutations in the parental RCC tissue and corresponding TOs. Common RCC genetic alterations, such as von Hippel-Lindau (VHL) and polybromo 1 (PBRM1) mutations, were detected in the RCC tumors and corresponding TOs (Fig. 4). The results suggested that patient-derived TOs resembled clinical tumors and that TOs could serve as a potential ex vivo model of RCC.

Patient-derived TOs respond differently to treatment with TKIs. To explore the utility of the TO cultures as potential tumor models for the evaluation of drug efficacy, the RCC SOC TKIs, sunitinib, axitinib, pazopanib, sorafenib and cabozantinib, were examined in four patient-derived RCC TO cultures (Fig. 5). To 


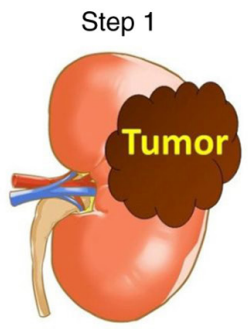

Collection of tumor sample
Step 2

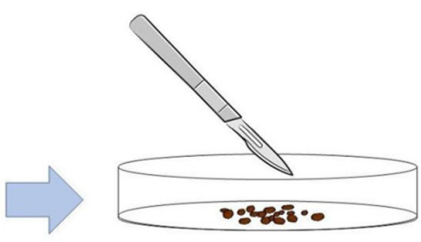

Tumor tissue dissociation

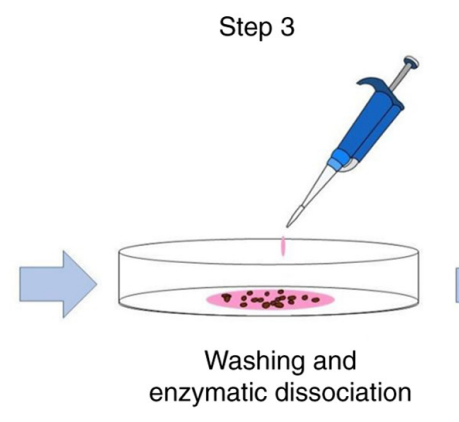

enzymatic dissociation

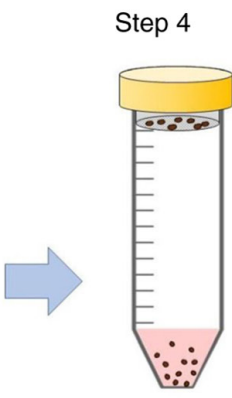

Strainer filtration

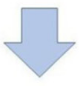

Step 5

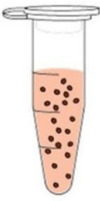

Mixing with matrigel

Figure 1. Method for the development of patient-derived tumor organoids. Step 1, tumor samples were collected from RCC tissue removed during radical nephrectomy or nephron-sparing surgery. Step 2, freshly resected tumor samples were placed in cold PBS and minced with scalpels. Step 3, the tumor tissue was incubated in collagenase solution at $37^{\circ} \mathrm{C}$ for $30 \mathrm{~min}$. Step 4, the dissociated tumor was passed through a $100-\mu \mathrm{m}$ cell strainer. Step 5, tumor cells were gently mixed with Matrigel. Step 6, the suspension of tumor cells in $20 \mu \mathrm{l}$ Matrigel was added to each well of a 24 -well plate and solidified at $37^{\circ} \mathrm{C}$ for $30 \mathrm{~min}$. Steps 7 and 8 , TO culture medium was added and tumor organoids were cultured at $37^{\circ} \mathrm{C}$. RCC, renal cell carcinoma; PBS, phosphate-buffered saline; TO, tumor organoid.
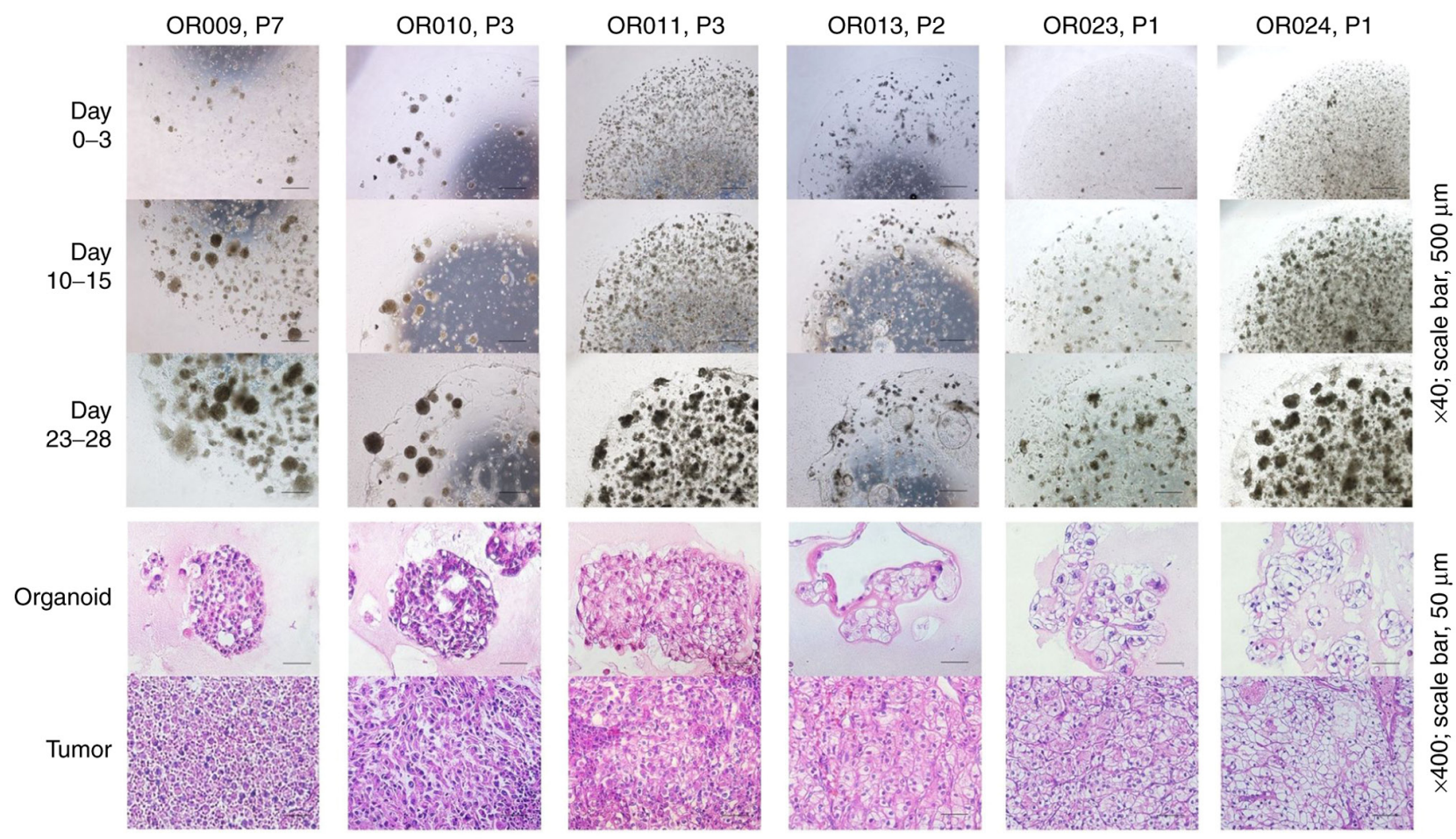

Figure 2. Representative images of RCC TOs. Bright-field images of patient-derived TOs in the early, middle, and late stages of TO culture with corresponding H\&E images of parental tumors and patient-derived TOs. RCC, renal cell carcinoma; TO, tumor organoid; H\&E, hematoxylin and eosin; P, passage number.

avoid the divergence of the parent tumor and TO lines due to potential clonal selection at late TO passages, each TO line was examined at an early passage. It was found that the $\mathrm{GI}_{50}$ values for the TKIs ranged from 2 to $50 \mu \mathrm{M}$ (Fig. 5). The TOs were defined as TKI-resistant when the $\mathrm{GI}_{50}$ of the drug was higher than the clinically relevant concentration (CRDC) of the same 

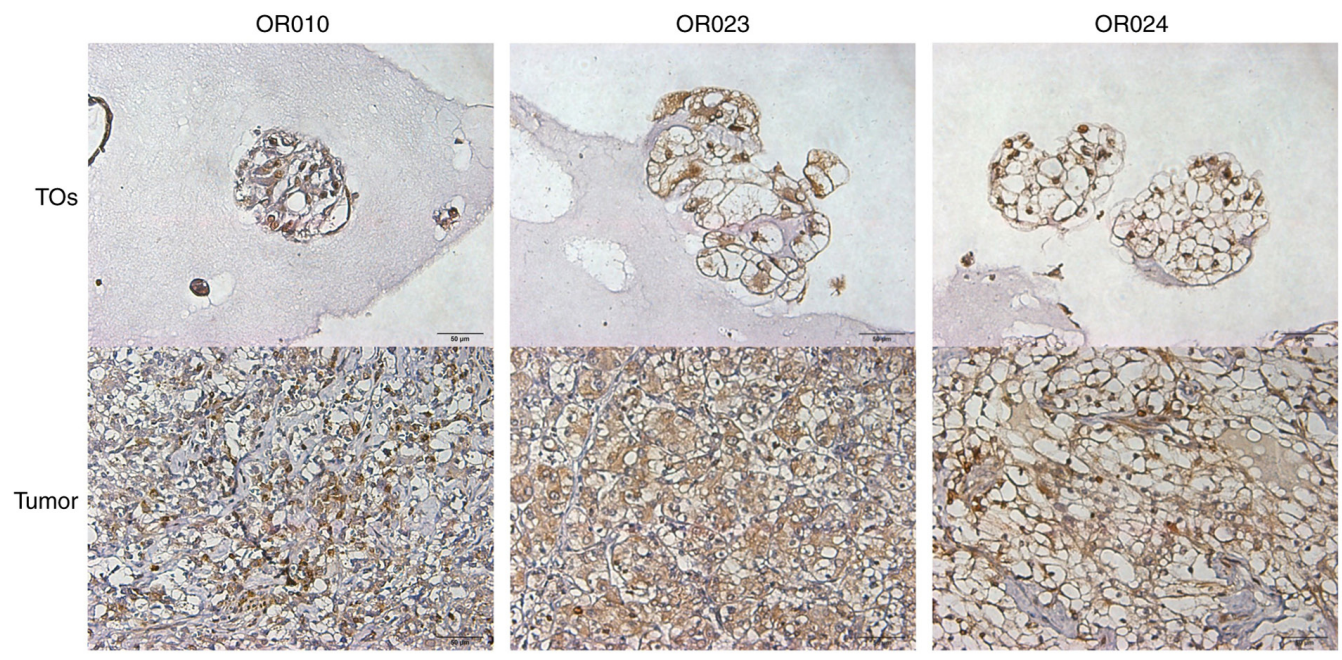

Figure 3. Representative images of Bcl-2 expression in primary tumor tissue and patient-derived TOs (magnification, $\mathrm{x} 400$; scale bar, $50 \mu \mathrm{m}$ ). Bcl-2 expression was detected by IHC staining. Bcl-2, B-cell lymphoma 2; IHC, immunohistochemistry; TOs, tumor organoids.

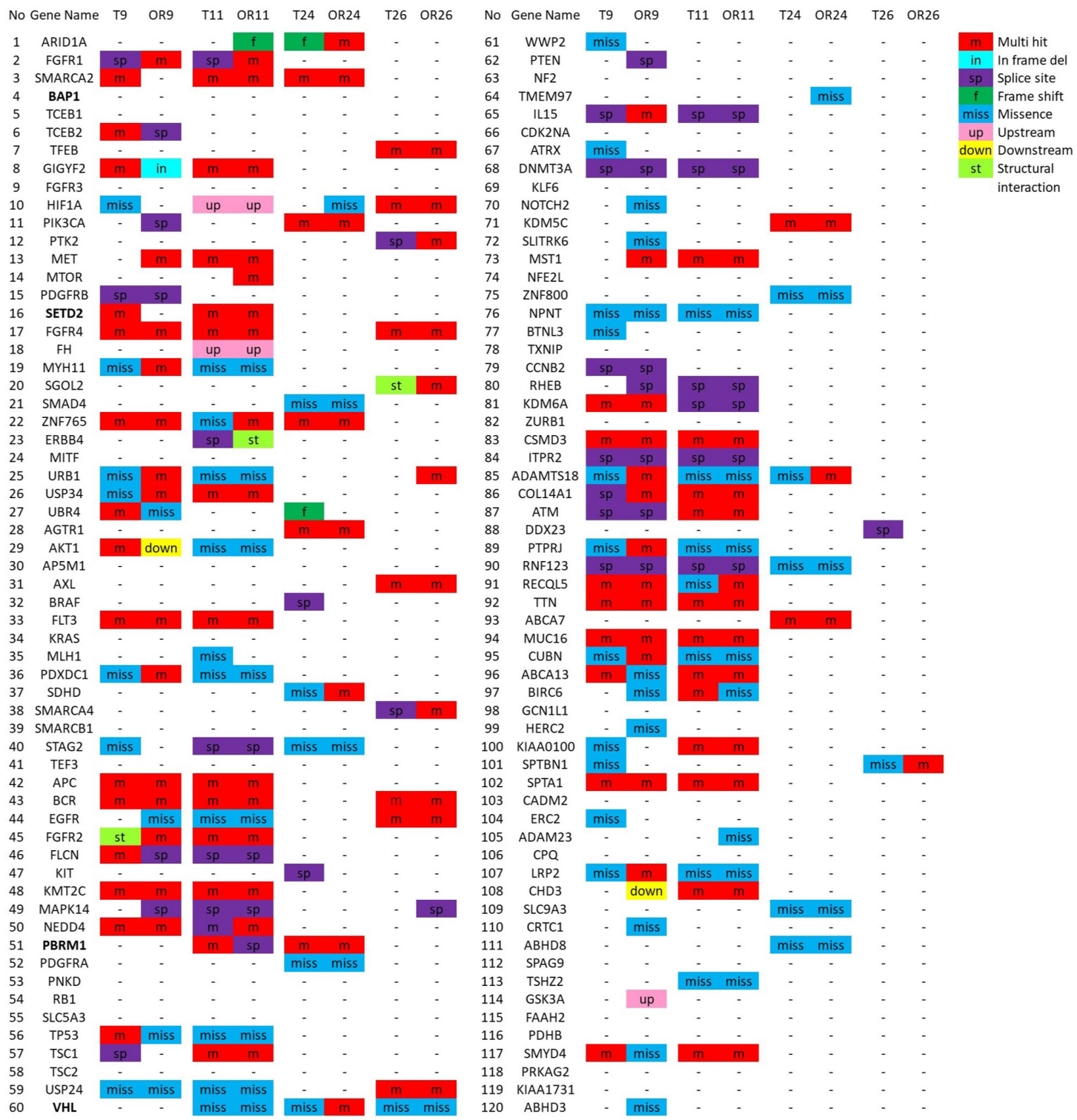

Figure 4. Summary of the genetic alterations identified in parental RCC and corresponding tumor organoids by whole-exome sequencing (cases OR009, OR011, OR024 and OR026). Representative genes known to be mutated in kidney cancer are shown in bold font. RCC, renal cell carcinoma; m, multi-hit; in, frame del; sp, splice site; f, frameshift; n, nonsense; miss, missence; up, upstream; down, downstream; st, structural interaction. RCC, renal cell carcinoma. 
A

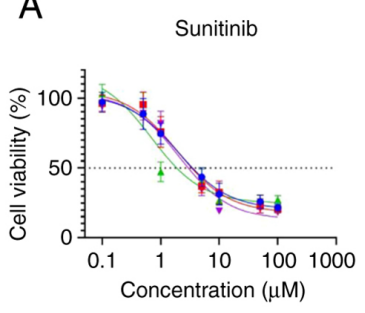

Pazopanib

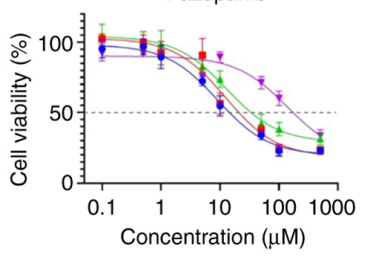

Cabozantinib

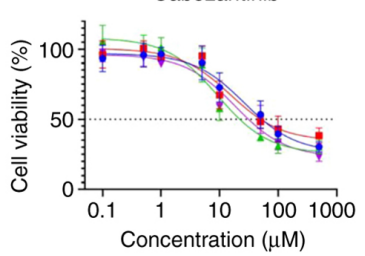

Axitinib

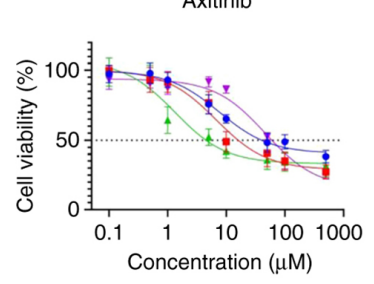

Sorafenib

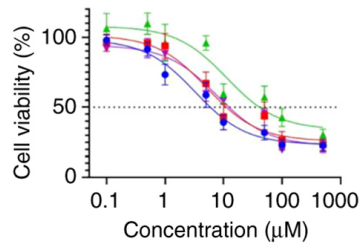

B
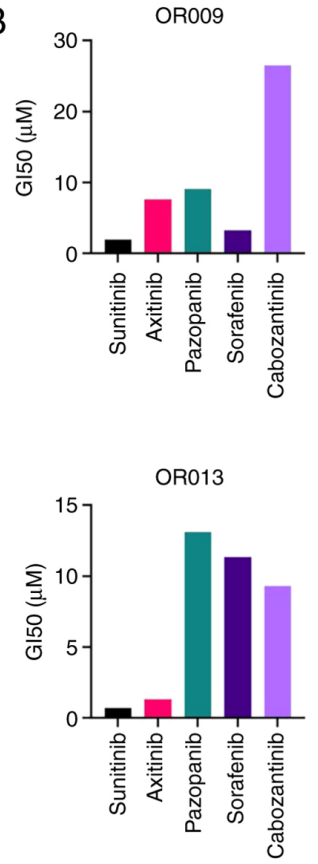

OR011

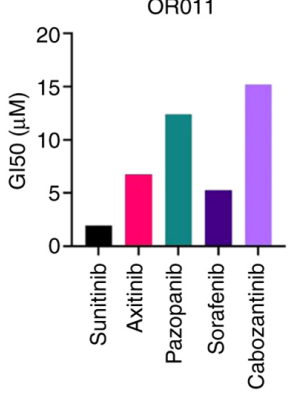

OR024

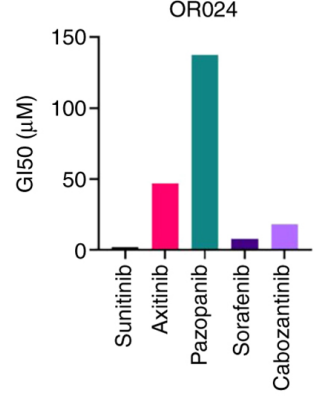

Figure 5. Analysis of the antitumor effects of TKIs in RCC tumor organoids. (A) Analysis of cancer cell viability using colorimetric CellTiter assay in OR009, OR011, OR013 and OR024 tumor organoids treated with the TKIs, sunitinib, axitinib, pazopanib, sorafenib and cabozantinib, for $72 \mathrm{~h}$. (B) GI ${ }_{50}$ for each TKI in RCC TO models using GraphPrism 8.0. TKI, tyrosine kinase inhibitor; RCC, renal cell carcinoma; TO, tumor organoid; $\mathrm{GI}_{50}$, drug concentration that inhibited the growth of cancer cells by $50 \%$.

drug. The CRDC is defined as the peak plasma concentration of a drug. Although all four TO cultures were resistant to sunitinib (CRDC, $200 \mathrm{nM}$ ), axitinib (CRDC, $160 \mathrm{nM}$ ) and cabozantinib (CRDC, $4 \mu \mathrm{M}$ ), it was found that three of the four TO cultures were sensitive to pazopanib (CRDC, $130 \mu \mathrm{M})$ and all TOs were sensitive to sorafenib (CRDC, $20 \mu \mathrm{M}$ ) (Fig. 5B) (15). The pazopanib $\mathrm{GI}_{50}$ was 8-10-fold lower than its CRDC in the OR009, OR011 and OR013 cases, whereas the sorafenib GI $_{50}$ was 2-5-fold lower than its CRDC in the same cases (Fig. 5B). These findings support the selection of pazopanib as the most effective drug for the OR009, OR011 and OR013 RCC cases, and sorafenib for case OR024.

\section{Discussion}

Oncologists aim to ensure that cancer patients are offered the optimal treatment options from the start in order to avoid delayed treatment, potential side-effects of ineffective treatments, and unnecessary expenses of therapies that do not benefit them.

The main objective of the present study was to develop and test patient-derived 3D TOs as a new personalized ex vivo tumor model to determine the most effective treatment for individual patients with RCC. The development of this approach includes the generation of TO culture from a patient's tumor biopsy specimen, development and execution of a functional personalized test of chemotherapeutic and targeted therapeutics in the laboratory, followed by the confirmation of the effectiveness of the predicted drug therapy in the clinic.

Usually, treatment with SOC drugs is initiated 30-90 days following the surgical resection of the tumor and the successful post-operative recovery of the patient. It provides a treatment-free period (30-90 days) to establish TOs, test SOC drugs in TOs, and identify the most effective therapeutic agent to begin the treatment of the patient. In metastatic cases, tumor core biopsy may be another source of tumor material to establish TOs. Tumor core biopsy can be performed at any time point during the patient's treatment, and it does not affect previous or current therapies administered to the patient. The method presented herein may prove to be useful for the selection of an effective therapy for patients who develop recurrence following radical nephrectomy, and for deciding on the second-line treatment for patients with RCC with disease progression during primary systemic therapy. This novel approach facilitates the efficient and rapid development of tumor-derived TOs with subsequent testing and identification of the optimal personalized treatment options for cancer patients.

Tumor-derived organoids have been developed in various cancer types, including lung (16), gastrointestinal (17), colon (18), prostate (8) and bladder (12) cancers. An initial report on the development of TOs from clear cell RCC (four cases) was published in 2019 (19). The present study selected the development of 3D TO models as the generation of $2 \mathrm{D}$ cell lines from primary tumors is inefficient, as it involves extensive adaptation and clonal selection for in vitro $2 \mathrm{D}$ culture conditions. As only rare clones are able to expand and can be maintained over several passages in $2 \mathrm{D}$ culture, the derived 2D cell lines may have undergone substantial genetic alterations and may no longer recapitulate the genetic heterogeneity of the original tumors. In the present study, a novel method for the efficient development of 3D TO cultures from freshly resected RCC tumor samples was developed. Importantly, this method allows the growth of RCC TOs 
using a simple culture medium without costly supplements (e.g., Noggin, gastrin, R-spondin, or Wnt3A) $(4,11,20,21)$. While previous TO studies have used Dulbecco's modified Eagle medium/nutrient mixture F-12 (DMEM/F12) medium with multiple supplements, the present study established TO cultures in $15(75 \%)$ of 20 RCC cases using hepatocyte culture medium. The quality of the tumor specimen is critical for TO development; however, further investigations are required in order to understand other factors affecting the successful establishment of TO culture.

Clinical tumors and patient-derived TO cultures share a number of features, including morphology, cell-cell interaction, signal transduction, gene and protein expression, hypoxia, differential zones of proliferation, $\mathrm{pH}$, drug response and resistance $(10,22)$. Consistent with previously published studies on other types of cancer $(8,12,15-17)$, the present study found that patient-derived RCC TO cultures possessed in vivo features of RCC clinical tumors, such as morphology, genetic alterations and the expression of the Bcl-2 anti-apoptotic protein, suggesting the potential use of the patient-derived RCC TO culture as a model for personalized medicine.

The evaluation of the therapeutic response of patient-derived TOs to antitumor drugs may be a potential approach for the development of personalized treatment. To the best of our knowledge, the present study is first to demonstrate the development of a method for selecting the optimal treatment options for patients with RCC by testing the SOC TKIs in patient-derived TO models. Although ICIs are becoming the mainstay of first-line treatment for metastatic RCC (23-25), the development of a TO assay for the testing of ICIs remains challenging due to the indirect effect of immunotherapeutic agents on cancer cells mediated by host immunity, which is difficult to recapitulate ex vivo. The development of TO-based assays for the testing of immuno-oncology therapeutics is a subject of future research studies. TKIs remain key targeted therapeutics in second-line RCC therapy (26). The selection of patients with RCC who are most likely to respond to TKI therapy is crucial for improving patient survival. The findings of the present study demonstrated a difference in response to TKI treatment in different ex vivo TO cultures established from RCC tumors. The present findings are consistent with those of previous studies that observed variations in drug sensitivity in different TO cultures (27-30). Herein, the ex vivo therapeutic response was defined as that with a $\mathrm{GI}_{50}$ lower than the CRDC (peak plasma concentration). This approach can be applied to evaluate the therapeutic response of targeted or chemotherapeutic drugs in various TO models. The ex vivo testing method used herein for the selection of the most effective TKI in patient-derived RCC TO models requires validation in clinical settings.

In conclusion, the present study demonstrated the development of a patient-derived TO culture and presented a novel approach for the ex vivo evaluation of the therapeutic response as a potential testing model for the selection of personalized therapy for patients with RCC.

\section{Acknowledgements}

Not applicable.

\section{Funding}

The present study was supported by a research grant from the Department of Urology, Division of Molecular Oncology, Niigata University Graduate School of Medical and Dental Sciences, Niigata, Japan (grant no. CH29017; August, 2018).

\section{Availability of data and materials}

The datasets generated and/or analyzed during the current study are available in the figshare repository, https://doi. org/10.6084/m9.figshare.14993409.v1.

\section{Authors' contributions}

AK, VB and YT conceived and designed the study. AK, TA, HK, YS, MM, VB, AU, KS and YT developed the study methodology. AK, TA, HK, YS and MM performed the experiments. AK, VB and YT analyzed and interpreted the data. AK, VB, AU and YT wrote, reviewed and revised the manuscript. YT provided administrative, technical, or material support. VB, $\mathrm{AU}, \mathrm{KS}$ and YT supervised the study. AK and VB confirm the authenticity of all the raw data. All authors have read and approved the final manuscript.

\section{Ethics approval and consent to participate}

The present study was approved by the Institutional Review Board of Niigata University (approval no. 2018-0254). Written informed consent was obtained from the patients.

\section{Patient consent for publication}

Not applicable.

\section{Competing interests}

The authors declare that they have no competing interests.

\section{References}

1. Kabaria R, Klaassen Z and Terris MK: Renal cell carcinoma: Links and risks. Int J Nephrol Renovasc Dis 9: 45-52, 2016.

2. Escudier B, Porta C, Schmidinger M, Rioux-Leclercq N, Bex A, Khoo V, Grünwald V, Gillessen S and Horwich A; ESMO Guidelines Committee. Electronic address: clinicalguidelines@ esmo.org: Renal cell carcinoma: ESMO clinical practice guidelines for diagnosis, treatment and follow-up. Ann Oncol 30: 706-720, 2019

3. Malone ER, Oliva M, Sabatini PJ, Stockley TL and Siu LL: Molecular profiling for precision cancer therapies. Genome Med 12: 8, 2020.

4. Fatehullah A, Tan SH and Barker N: Organoids as an in vitro model of human development and disease. Nat Cell Biol 18: 246-254, 2016.

5. Clevers H: Modeling development and disease with organoids. Cell 165: 1586-1597, 2016.

6. Rookmaaker MB, Schutgens F, Verhaar MC and Clevers H: Development and application of human adult stem or progenitor cell organoids. Nat Rev Nephrol 11: 546-554, 2015.

7. Kapałczyńska M, Kolenda T, Przybyła W, Zajączkowska M, Teresiak A, Filas V, Ibbs M, Bliźniak R, Łuczewski $Ł$ and Lamperska $\mathrm{K}: 2 \mathrm{D}$ and $3 \mathrm{D}$ cell cultures-a comparison of different types of cancer cell cultures. Arch Med Sci 4: 910-919, 2018. 
8. Broutier L, Mastrogiovanni G, Verstegen MM, Francies HE, Gavarró LM, Bradshaw CR, Allen GE, Arnes-Benito R, Sidorova O, Gaspersz MP, et al: Human primary liver cancer-derived organoid cultures for disease modeling and drug screening. Nat Med 12: 1424-1435, 2017.

9. Gao D, Vela I, Sboner A, Iaquinta PJ, Karthaus WR, Gopalan A, Dowling C, Wanjala JN, Undvall EA, Arora VK, et al: Organoid cultures derived from patients with advanced prostate cancer. Cell 159: 176-187, 2014.

10. Ganesh K, Wu C, O'Rourke KP, Szeglin BC, Zheng Y, Sauvé CG, Adileh M, Wasserman I, Marco MR, Kim AS, et al: A rectal cancer organoid platform to study individual responses to chemoradiation. Nat Med 25: 1607-1614, 2019.

11. Romero-Calvo I, Weber CR, Ray M, Brown M, Kirby K, Nandi RK, Long TM, Sparrow SM, Ugolkov A, Qiang W, et al: Human organoids share structural and genetic features with primary pancreatic adenocarcinoma tumors. Mol Cancer Res 17: 70-83, 2019.

12. Saito Y, Muramatsu T, Kanai Y, Ojima H, Sukeda A, Hiraoka N, Arai E, Sugiyama Y, Matsuzaki J, Uchida R, et al: Establishment of patient-derived organoids and drug screening for biliary tract carcinoma. Cell Rep 27: 1265-1276.e4, 2019.

13. Lee SH, Hu W, Matulay JT, Silva MV, Owczarek TB, Kim K, Chua CW, Barlow LJ, Kandoth C, Williams AB, et al: Tumor evolution and drug response in patient-derived organoid models of bladder cancer. Cell 173: 515-528.e17, 2018.

14. Bilim V, Yuuki K, Itoi T, Muto A, Kato T, Nagaoka A, Motoyama T and Tomita Y: Double inhibition of XIAP and Bcl-2 axis is beneficial for retrieving sensitivity of renal cell cancer to apoptosis. Br J Cancer 98: 941-949, 2008.

15. Liston DR and Davis M: Clinically relevant concentrations of anticancer drugs: A guide for nonclinical studies. Clin Cancer Res 23: 3489-3498, 2017.

16. Kim M, Mun H, Sung CO, Cho EJ, Jeon HJ, Chun SM, Jung DJ, Shin TH, Jeong GS, Kim DK, et al: Patient-derived lung cancer organoids as in vitro cancer models for therapeutic screening. Nat Commun 10: 3991, 2019.

17. Aberle MR, Burkhart RA, Tiriac H, Olde Damink SW Dejong $\mathrm{CH}$, Tuveson DA and van Dam RM: Patient-derived organoid models help define personalized management of gastrointestinal cancer. Br J Surg 105: e48-e60, 2018.

18. van de Wetering M, Francies HE, Francis JM, Bounova G, Iorio F, Pronk A, van Houdt W, van Gorp J, Taylor-Weiner A, Kester L, et al: Prospective derivation of a living organoid biobank of colorectal cancer patients. Cell 161: 933-945, 2015.

19. Grassi L, Alfonsi R, Francescangeli F, Signore M, De Angelis ML Addario A, Costantini M, Flex E, Ciolfi A, Pizzi S, et al: Organoids as a new model for improving regenerative medicine and cancer personalized therapy in renal diseases. Cell Death Dis 10: 201, 2019.
20. Neal JT, Li X, Zhu J, Giangarra V, Grzeskowiak CL, Ju J, Liu IH, Chiou SH, Salahudeen AA, Smith AR, et al: Organoid modeling of the tumor immune microenvironment. Cell 175: 1972-1988. e16, 2018.

21. Vlachogiannis G, Hedayat S, Vatsiou A, Jamin Y, Fernández-Mateos J, Khan K, Lampis A, Eason K, Huntingford I, Burke R, et al: Patient-derived organoids model treatment response of metastatic gastrointestinal cancers. Science 359: 920-926, 2018

22. Bian S, Repic M, Guo Z, Kavirayani A, Burkard T, Bagley JA, Krauditsch C and Knoblich JA: Author correction: Genetically engineered cerebral organoids model brain tumor formation. Nat Methods 15: 748, 2018.

23. Motzer RJ, Penkov K, Haanen J, Rini B, Albiges L, Campbell MT, Venugopal B, Kollmannsberger C, Negrier S, Uemura M, et al: Avelumab plus Axitinib versus Sunitinib for advanced renal-cell carcinoma. N Engl J Med 380: 1103-1115, 2019.

24. Rini BI, Plimack ER, Stus V, Gafanov R, Hawkins R, Nosov D, Pouliot F, Alekseev B, Soulières D, Melichar B, et al: KEYNOTE-426 investigators. Pembrolizumab plus Axitinib versus Sunitinib for advanced renal-cell carcinoma. N Engl J Med 380: 1116-1127, 2019.

25. Motzer RJ, Tannir NM, McDermott DF, Arén Frontera O, Melichar B, Choueiri TK, Plimack ER, Barthélémy P, Porta C, George S, et al: CheckMate 214 investigators. Nivolumab plus Ipilimumab versus Sunitinib in advanced renal-cell carcinoma. N Engl J Med 378: 1277-1290, 2018.

26. Zerdes I, Tolia M, Tsoukalas N, Mitsis M, Kardamakis D, Pistevou-Gombaki K, Tsekeris P and Kyrgias G: Systemic therapy of metastatic renal cell carcinoma: Review of the current literature. Urologia 86: 3-8, 2019.

27. Liu C, Qin T, Huang Y, Li Y, Chen G and Sun C: Drug screening model meets cancer organoid technology. Transl Oncol 13: $100840,2020$.

28. Li M and Izpisua Belmonte JC: Organoids-preclinical models of human disease. N Engl J Med 380: 569-579, 2019.

29. Bleijs M, van de Wetering M, Clevers H and Drost J: Xenograft and organoid model systems in cancer research. EMBO J 38: e101654, 2019.

30. Drost J and Clevers H: Organoids in cancer research. Nat Rev Cancer 18: 407-418, 2018

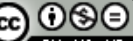

This work is licensed under a Creative Commons Attribution-NonCommercial-NoDerivatives 4.0 International (CC BY-NC-ND 4.0) License. 